\title{
OVERVIEW OF MALARIA PARASITE AND ITS PREVENTION IN INDIA
}

\author{
Adil Raza ${ }^{1}$, Megha Chaudhary ${ }^{2}$, Sonika Devi ${ }^{3}$ \\ ${ }^{1}$ Assistant Professor, NIMS University, Paramedical College, Jaipur \\ ${ }^{2}$ Assistant Professor, NIMS University, Paramedical College, Jaipur \\ ${ }^{3}$ MSc. Medical Microbiology
}

Article Info: Received 30 January 2019; Accepted 20 February. 2019

Cite this article as: Raza, A., Chaudhary, M., \& Devi, S. (2019). OVERVIEW OF MALARIA PARASITE AND ITS PREVENTION IN INDIA. Journal of Biomedical and Pharmaceutical Research, 8(1).

DOI: https://doi.org/10.32553/jbpr.v8i1.575

Address for Correspondence: Adil Raza, Assistant Professor, NIMS University, Paramedical College, Jaipur Conflict of interest statement: No conflict of interest

\section{ABSTRACT:}

Background: Malaria is a systematic disease caused by a parasite called Plasmodium which is transmitted into the human blood via female Anopheles mosquito. Malaria in humans is caused by four species of protozoan parasites of the genus Plasmodium: $P$. falciparum, $P$. vivax, $P$. ovale, and $P$. malariae. The parasite enters the human body through a mosquito bite and travel to the very crucial organ, the liver, where they multiply and come back to the bloodstream and destroy red blood cells. Malaria causes symptoms that typically include fever, tiredness, vomiting, and headaches. In severe cases it can cause yellow skin, seizures, coma, or death. Symptoms usually begin ten to fifteen days after being bitten by an infected mosquito. In those who have recently survived an infection, reinfection usually causes milder symptoms.

Objectives: Isolation of different species of malaria parasites. The prevalence of malaria parasite in India.

Methods: The procedure follows these steps: collection of peripheral blood, staining of smear with Leishman's stain and examination of red blood cells for malaria parasites under the microscope.

Results: We observed the plasmodium species in peripheral blood smear.

Conclusion: Worldwide, the number of cases of malaria caused by Plasmodium falciparum, the most dangerous species of the parasite, is on the rise.

\section{HISTORY}

Our understanding of the malaria parasites begins in 1880 with the discovery of the parasites in the blood of malaria patients by Alphonse Laveran. India's expansive geography and diverse climate supports ideal environments for sustaining malaria parasites and their vectors. The two major human malaria species in India are Plasmodium falciparum and $P$. vivax; $P$. malariae has been in the eastern India state of Orissa, ${ }^{1}$ while $P$. ovale appears to be extremely rare if not absentreported. Intriguingly, the two major infecting species vary in proportion across India. For example, the southern state of Tamil 
Nadu suffers from $P$. vivax, $P$. falciparum is the dominant parasite in Orissa, and mixed-species infections are prevalent in the west (e.g., Gujarat state) ${ }^{2}$. As the second most populous country in the world, with a population exceeding one billion people, India's public health system faces many challenges including implementation of surveillance programs to accurately estimate and control the national malaria burden. Historically, the highest incidence of malaria in India occurred in the 1950s, with an estimated 75 million cases and 0.8 million deaths per year (World Health Organization, Country Office for India). The launch of the National Malaria Control Program (NMCP) in 1953 resulted in a significant decline in the number of reported cases to $<50,000$ and no reported mortality, by 1961. Despite its near elimination in the mid1960's, malaria resurged to 6.45 million cases in 1976. Since then, confirmed cases have gradually decreased to 1.6 million cases and $\sim 1,100$ deaths in 2009. Recently, it has been suggested that the malaria incidence is between 9 to 50 times greater than reported (reviewed in Hay et al., 2010), with a 13-fold underestimation of malaria-related mortality (Dhingra et al., 2010). Such claims reinforce the need for robust and comprehensive epidemiological surveillance studies across the country (Singh et al., 2009) to determine the actual burden.

\section{INTRODUCTION}

Malaria is a mosquito-borne infectious disease affecting humans and other animals caused by single-celled microorganisms belonging to the Plasmodium group. The mosquito bite introduces the parasites from the mosquito's saliva into a person's blood.

The parasites travel to the liver where they mature and reproduce. There are more than 100 species of Plasmodium, which can infect many animal species such as reptiles, birds, and various mammals. Four species of Plasmodium have long been recognized to infect humans in nature. The genus Plasmodium is classified into two subgenera: $P$. vivax, $P$. malariae and $P$. ovale belong to the subgenus Plasmodium while $P$. falciparum belongs to the subgenus Laverania because it differs in a number of aspects from the other three species. P. vivax, P. malariae and P. ovale are closely related to other primate malaria parasites. $P$. falciparum is more related to bird malaria parasites and appears to be a recent parasite of humans, in evolutionary terms. Perhaps for this reason, falciparum infection causes the most severe form of malaria and is responsible for nearly all fatal cases. $P$. knowlesi, a parasite of long-tailed Macaque monkeys may also affect man $^{3}$

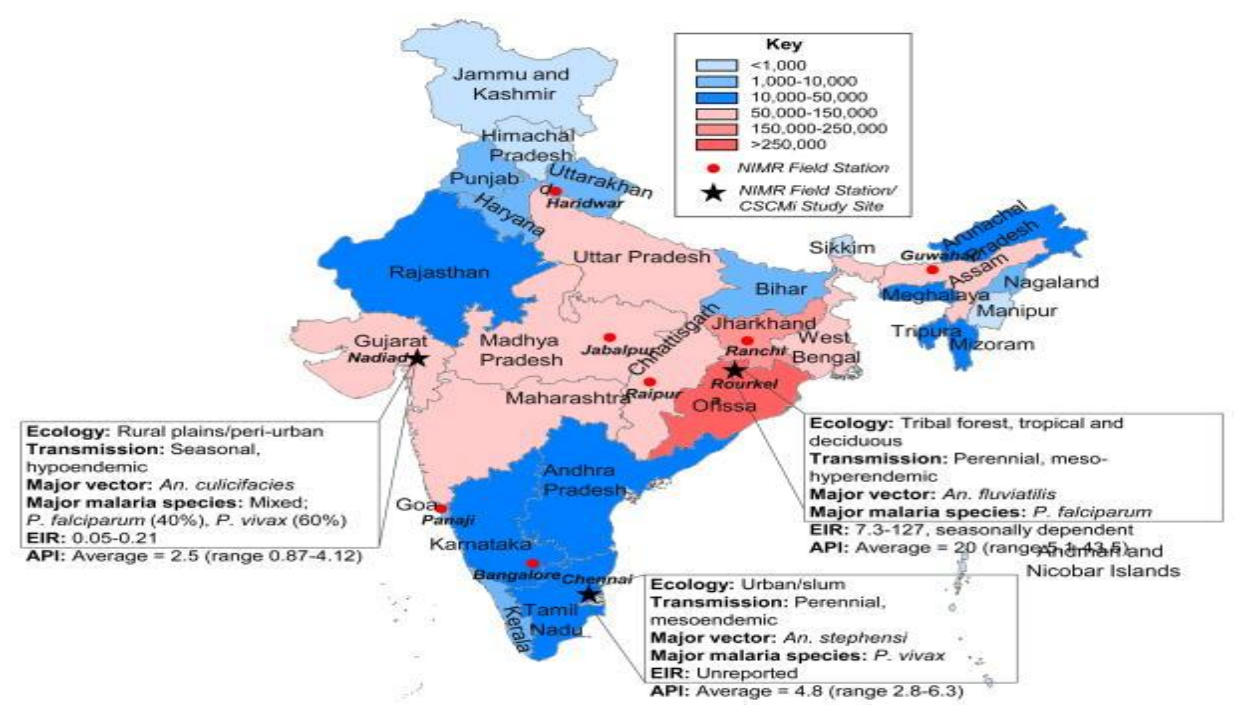




\section{MATERIAL AND METHODS:}

Preparation of the smear: Use universal precautions while preparing the smears for malarial parasites - use gloves; use only disposable needles/lancets; wash hands; handle and dispose the sharp instruments and other materials contaminated with blood carefully to avoid injury.

- Hold the third finger of the left hand and wipe its tip with spirit/Savlon swab; allow to dry Prick the finger with disposable needle/lancet; allow the blood to ooze out.

- Take a clean glass slide.

- Take 3 drops of Blood $1 \mathrm{~cm}$ from the edge of the slide take another drop of blood one $\mathrm{cm}$ from the first drop of blood.

- Take another clean slide with smooth edges and use it as a spreader and make thick and thin smears.

Allow it to dry Slide number can be marked on the thin smear with a lead pencil.

\section{Blood collection for thin or thick blood film.}

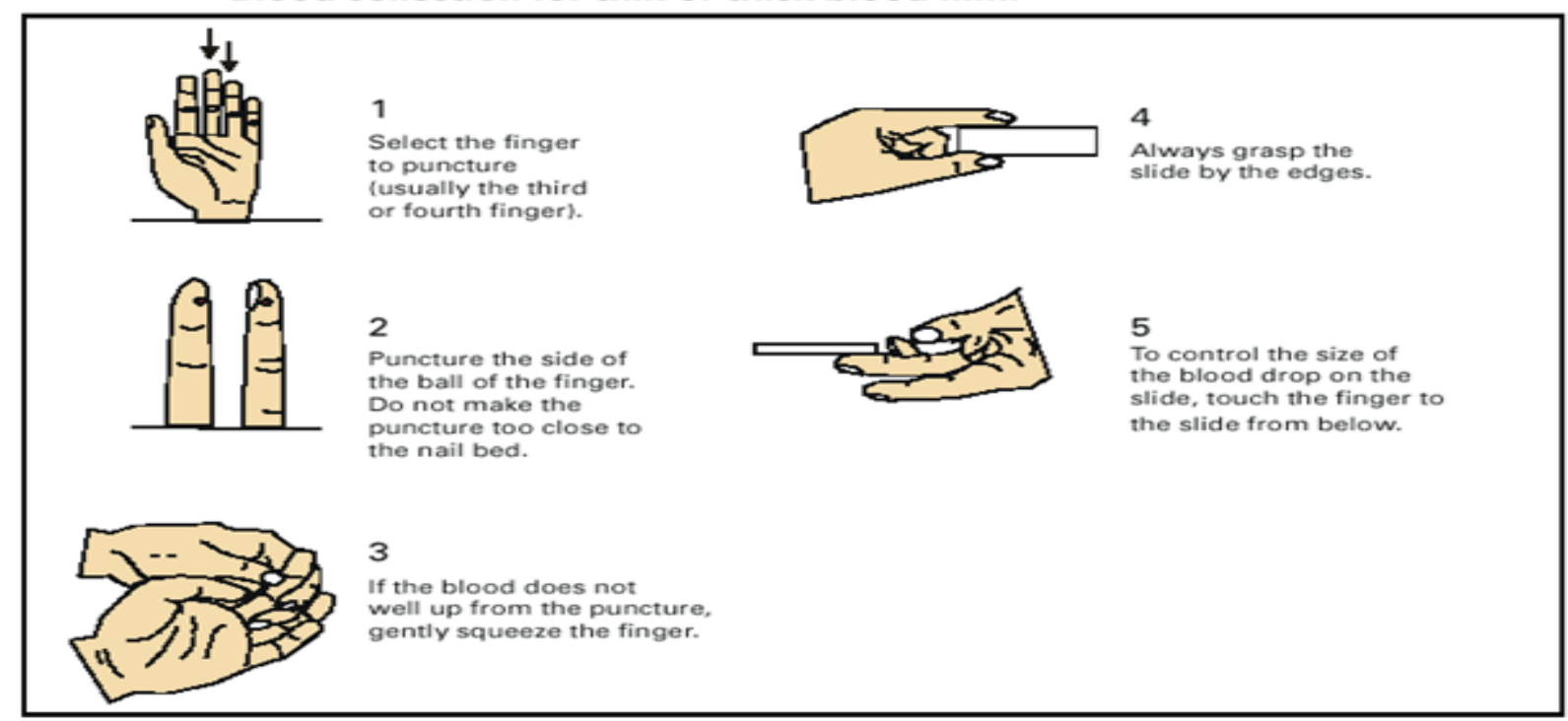

\section{Leishman's stain:}

- $\quad$ Add 7-8 drops of the stain and leave for 12 minutes.

- $\quad$ Then add 12-15 drops of buffered distilled water, mix thoroughly, leave for $4-8$ minutes.

- Then wash off with clean water, drain, dry and examine.

\section{LIFE CYCLE OF MALARIA PARASITE}

The malaria parasite has a complex, multistage life cycle occurring within two living beings, the vector mosquitoes and the vertebrate hosts. The survival and development of the parasite within the invertebrate and vertebrate hosts, in intracellular and extracellular environments, is made possible by a toolkit of more than 5,000 genes and their specialized proteins that help the parasite to invade and grow within multiple cell types and to evade host immune responses.[2,3] The parasite passes through several stages of development such as the sporozoites (Gr. Sporos = seeds; the infectious form injected by the mosquito), merozoites (Gr. Meros = piece; the stage invading the erythrocytes), trophozoites (Gr. Trophes $=$ nourishment; the form multiplying in erythrocytes), and gametocytes (sexual stages) and all these stages have their own unique shapes and structures and protein complements. The surface proteins and metabolic pathways keep changing during these different stages, that help the parasite to evade the immune clearance, while also creating problems for the development of drugs and vaccines.[3] 
Adil Raza et al, Journal of Biomedical and Pharmaceutical Research

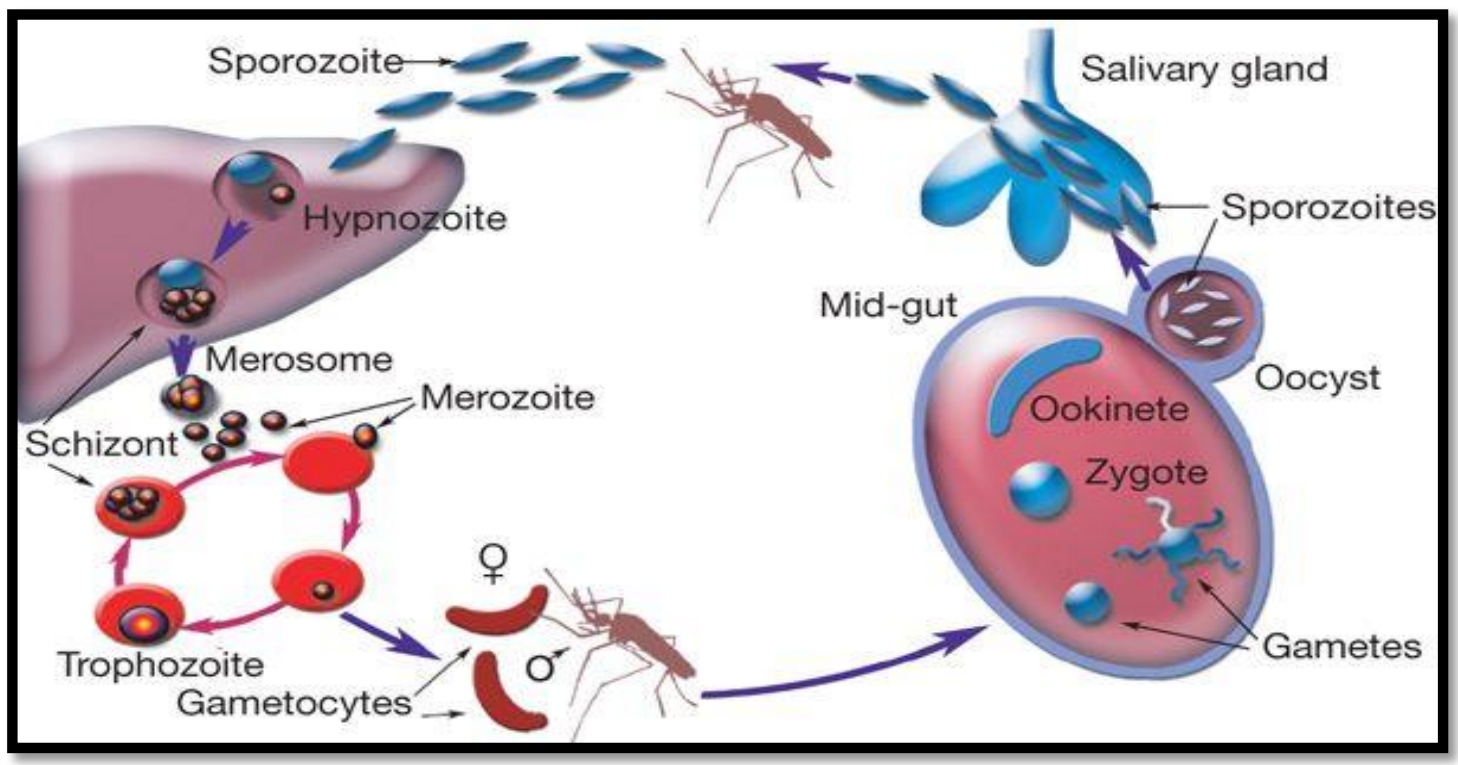

\section{P. malariae}

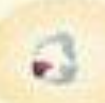

ring form

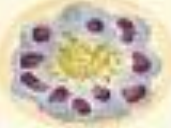

macure sehtront

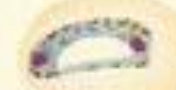

early band form

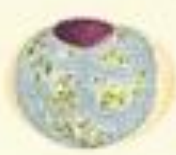

female gamerocyre

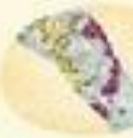

band form

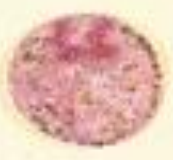

male gamerocyte

early schizont martipe sefhizont
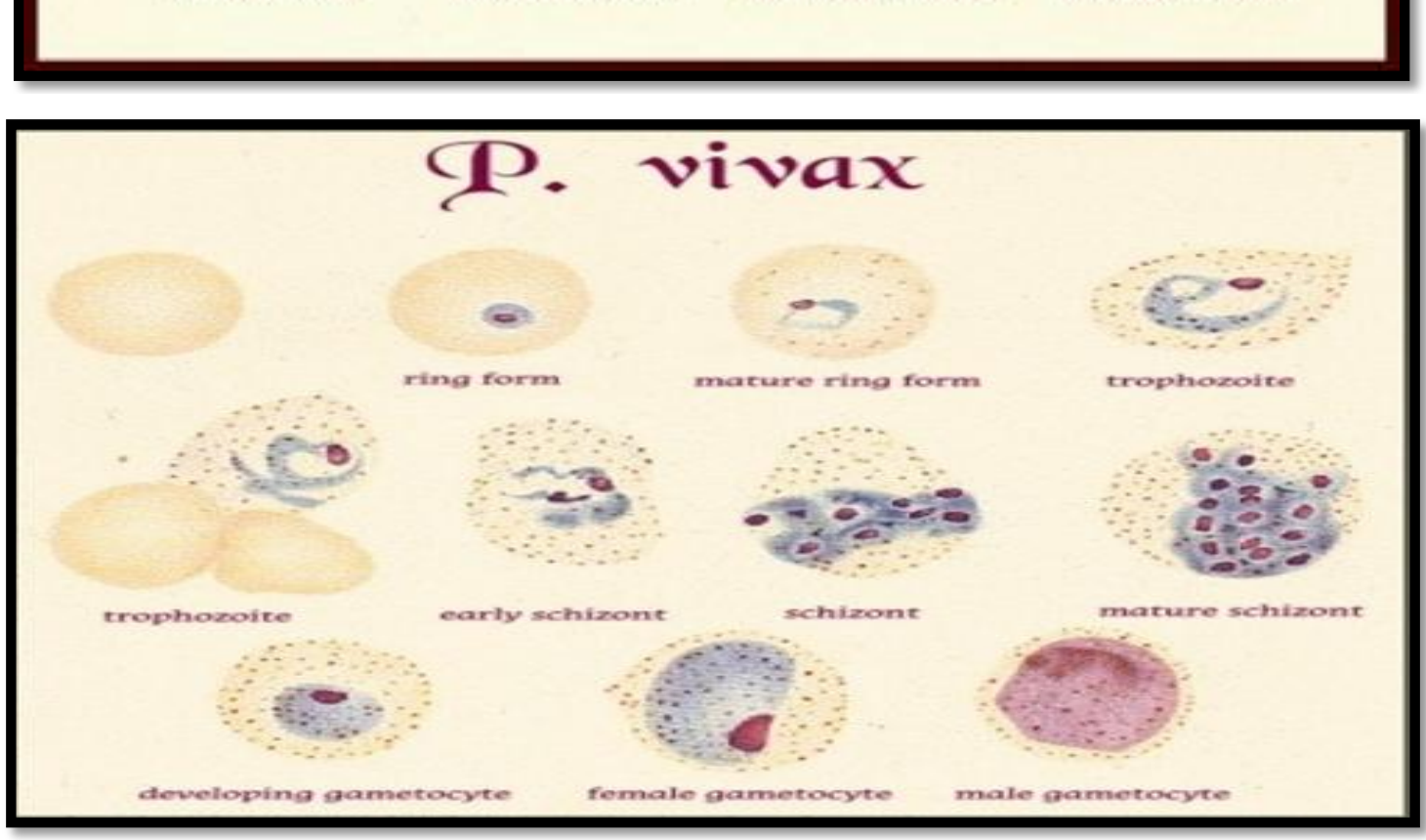

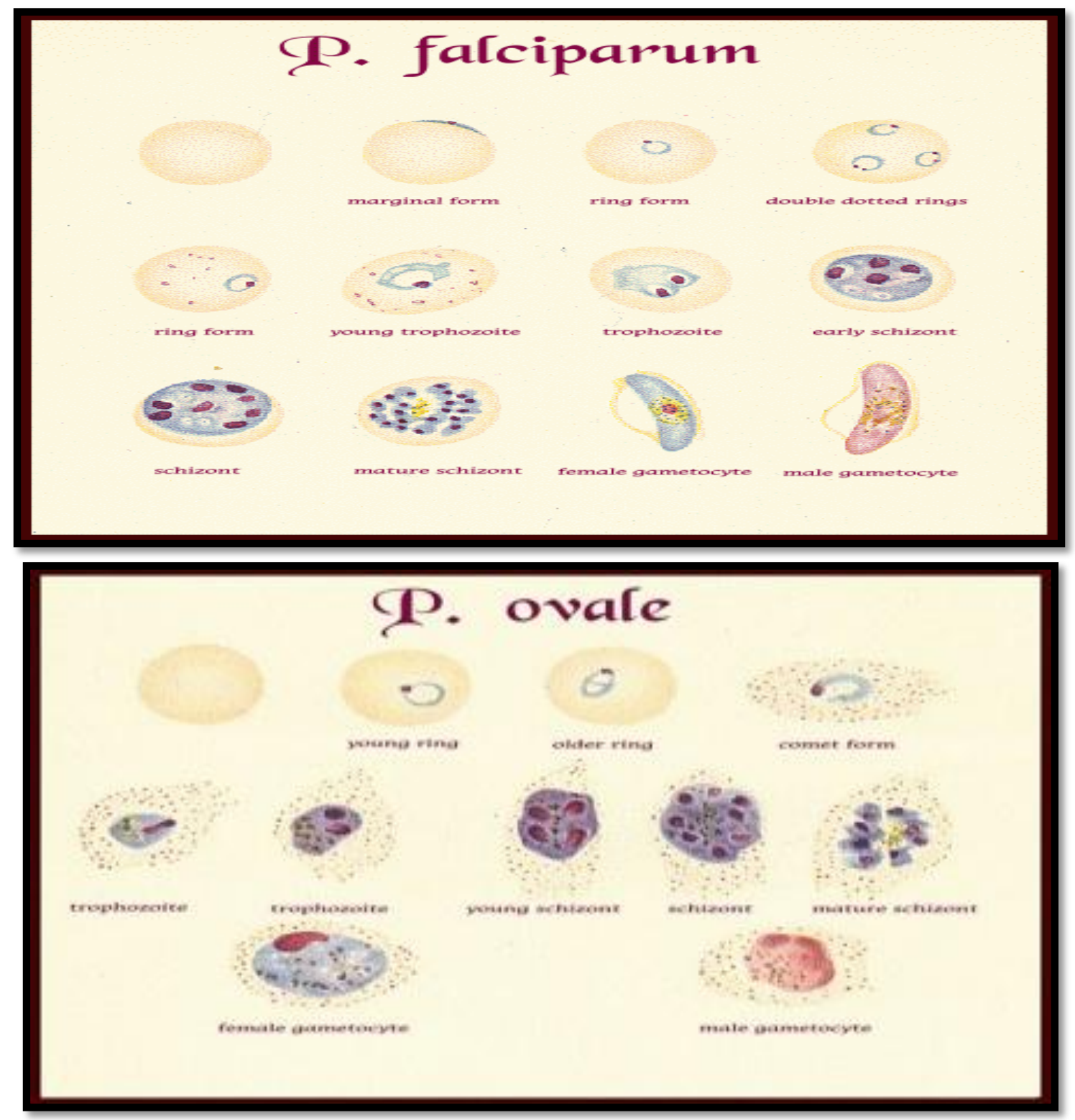

\section{PREVENTION}

Malaria is a preventable and treatable disease. Interventions to prevent malaria include: Vector control: sleeping under insecticidetreated nets (ITNs), indoor residual spraying (IRS) and in some specific settings, larval control. Intermittent preventive treatment for pregnant women and infants and seasonal chemoprophylaxis for children 1-5 years of age. Diagnosis and treatment of malaria. Awareness of risk - find out whether you're at risk of getting malaria. Bite prevention - avoid mosquito bites by using insect repellent, covering your arms and legs, and using a mosquito net. Check whether you need to take malaria prevention tablets - if you do, make sure you take the right antimalarial tablets at the right dose, and finish the course. Diagnosis - seek immediate medical advice if you have malaria symptoms, including up to a year after you return from travelling. To avoid being bitten: Stay somewhere that has effective air conditioning and screening on doors and windows. If this isn't possible, make sure doors and windows close properly. If you're not sleeping in an air-conditioned room, sleep under an intact mosquito net that's been treated with insecticide. Use insect repellent on your skin and in sleeping environments. 
Remember to reapply it frequently. The most effective repellents contain diethyltoluamide (DEET) and are available in sprays, roll-ons, sticks and creams. Wear light, loose-fitting trousers rather than shorts, and wear shirts with long sleeves. This is particularly important during early evening and at night, when mosquitoes prefer to feed. When taking antimalarial medication: make sure you get the right antimalarial tablets before you go - check with your GP or pharmacist if you're unsure follow the instructions included with your tablets carefully depending on the type you're taking, continue to take your tablets for up to 4 weeks after returning from your trip to cover the incubation period of the disease

\section{REFERNCE}

1. Sharma SK, Tyagi PK, Padhan K, Upadhyay AK, Haque MA, Nanda N, Joshi H, Biswas S, Adak T, Das BS, Chauhan VS, Chitnis CE, Subbarao SK. Epidemiology of malaria transmission in forest and plain ecotype villages in Sundargarh District, Orissa, India. Trans R Soc Trop Med Hyg. 2006; 100(10):917-925. [PubMed]

2. Singh N, Kataria $O$, Singh MP. The changing dynamics of Plasmodium vivax and $P$. falciparum in central India: trends over a 27-year period (19752002) Vector Borne Zoonotic Dis. 2004; 4(3):239-248. [PubMed]

3. Paniker's textbook of medical parasitology $8^{\text {th }}$ edition. 2018.
4. Hay SI, Gething PW, Snow RW. India's invisible malaria burden. Lancet. 2010; 376:1716-1717.[PMC free article] [PubMed]

5. Dhingra $N$, Jha $P$, Sharma VP, Cohen AA, Jotkar RM, Rodriguez PS, Bassani DG, Suraweera W, Laxminarayan R, Peto R. Adult and child malaria mortality in India. Lancet. 2010;376:1768-1774.[PMC free article] [PubMed]

6. Singh N, Kataria O, Singh MP. The changing dynamics of Plasmodium vivax and $P$. falciparum in central India: trends over a 27-year period (19752002) Vector Borne Zoonotic Dis. 2004; 4(3):239-248. [PubMed]

7. "CDC - Malaria Parasites - About". CDC: Malaria. U.S. Centers for Disease Control and Prevention. Retrieved 28 December 2015.

8. Brian M. Greenwood, David A. Fidock, Dennis E. Kyle, Stefan H.I. Kappe, Pedro L. Alonso, Frank H. Collins, Patrick E. Duffy. Malaria: progress, perils, and prospects for eradication. J. Clin. Invest. 2008;118:12661276. doi:10.1172/JCl33996 Full Text at http://www.jci.org/articles/view/33996 /files/pdf

9. Laurence Floren, Michael P. Washburn, J. Dale Raine et al. A proteomic view of the Plasmodium falciparum life cycle Nature October 2002;419:520-526. Full text athttp://www.nature.com/nature/ journal/v419/n6906/pdf/nature01107.pdf 